\title{
COMPRENSIÓN TEÓRICA Y PROCESO METODOLÓGICO DE LA INVESTIGACIÓN CUALITATIVA
}

\author{
UNDERSTANDING THEORETICAL AND METHODOLOGICAL PROCESS OF \\ QUALITATIVE RESEARCH
}

Elmina Matilde Rivadeneira Rodríguez

\begin{abstract}
RESUMEN
T a presente investigación tiene por objetivo general analizar los lineamientos teóricos Lpara comprender el proceso evolutivo que garantiza la metodología de la investigación cualitativa. Los métodos cualitativos estudian los hechos observables, en los que la creatividad y motivación del investigador permiten que sea considerado como sujeto y miembro principal de la investigación cualitativa. Con ello permite que sus interpretaciones sean flexibles, dinámicas, para resolver los problemas proactivamente. En la actualidad, el problema que enfrenta la investigación cualitativa es la falta de comprensión teórica, epistemológica-metodológica. La confusión conceptual de conocimiento y de ciencia. Sin embargo, en las últimas décadas, sobre todo en las Ciencias Humanas, han surgido nuevos métodos, estrategias, procedimientos, técnicas e instrumentos para abordar y enfrentar esta compleja realidad.
\end{abstract}

Palabras clave: comprensión, investigación cualitativa integradora holística

\section{ABSTRACT}

This research was overall goal: "To analyze the theoretical basis for understanding the evolutionary process that ensures qualitative research methodology." When referring to qualitative methods such study observable facts, where creativity, motivation research allows it to be considered as subject and leading member of the qualitative research, thereby allowing us that their interpretations are flexible, dynamic to solve problems proactively. At the present time the problem facing qualitative research is the lack of theoretical understanding epistemological-methodological. The conceptual confusion of knowledge and science. No clutch in recent decades, there have been new methods, 
strategies, procedures, techniques and instruments, especially in the human sciences, to address and tackle this complex reality.

Keywords: Understanding, research, qualitative integration holistic

\section{INTRODUCCIÓN}

La comprensión teórica en relación con la investigación cualitativa se refiere a la integración de un todo y sus partes. En el primer plano, se realiza una revisión de las aportaciones teóricas en relación con la comprensión de la investigación cualitativa, destacando la importancia del paradigma posmodernista. En segundo plano, se presentan las cualidades del enfoque cualitativo, los métodos cualitativos y los tipos de investigación cualitativa. Por último, se enuncia la propuesta de una metodología integradora holística para poder conseguir el estudio del fenómeno de un todo y sus partes.

La metodología integradora holística pretende desarrollar la relación entre el todo y las partes: el ser humano-investigador: "Un todo físico, químico, biológico, psicológico, social, cultural, espiritual”. Diálogo holístico: (ser y existencia). La participación activa y democrática. Respetar las dos grandes tradiciones filosóficas predominantes: realismo e idealismo. Recoger toda la información necesaria y suficiente para alcanzar esos objetivos o solucionar ese problema. Estructurar esa información en un todo coherente y lógico, es decir, idear una estructura lógica, un modelo o una teoría que integre esa información (Martínez, 2006).

\section{PROBLEMA}

Al desarrollar la investigación se busca responder la siguiente interrogante principal:

¿Cuáles son los lineamientos teóricos que propician la comprensión del proceso evolutivo de la metodología integradora holística de la investigación cualitativa?

Esto conlleva a responder las siguientes interrogantes secundarias:

- ¿Cuáles son los principales lineamientos teóricos que permiten la comprensión del proceso evolutivo de la metodología de la investigación cualitativa?

- ¿Por qué es importante conocer los lineamientos teóricos que garantizan el proceso evolutivo de la metodología de la investigación que permitan comprender el proceso evolutivo de la investigación cualitativa?

- ¿Cuáles son los lineamientos teóricos que permiten desarrollar una metodología integradora holística para garantizar el proceso evolutivo y la comprensión de la investigación cualitativa? 


\section{Objetivo general}

Analizar los lineamientos teóricos de la investigación cualitativa para propiciar la comprensión del proceso evolutivo de la metodología de la integradora holística.

\section{Objetivos específicos}

- Recopilar los lineamientos teóricos para garantizar la comprensión del proceso evolutivo de la metodología de la investigación cualitativa.

- Explicar los lineamientos teóricos para garantizar el proceso evolutivo de la metodología investigación cualitativa.

- Establecer las acciones de una metodología integradora holística apoyándose en los fundamentos teóricos y la comprensión para el estudio del fenómeno científico.

\section{METODOLOGÍA}

La metodología empleada se basó en la consulta bibliográfica de las bibliotecas del campus universitario, y artículos publicados en internet como recursos complementarios. La metodología es de carácter eminentemente teórico porque su objetivo es analizar los lineamientos teóricos para comprender el proceso evolutivo que garantiza el proceso de la metodología cualitativa. Respecto a su diseño de tipo documental, en esta investigación se utilizó la técnica de análisis de contenido, aplicable a discursos, información, mensajes, textos, imágenes, etc.

\section{REVISIÓN TEÓRICA}

Desde diversas perspectivas, el sustento epistemológico de la investigación cualitativa es un asunto muy discutido, según las formas de definir la realidad, al investigador, la investigación, el método y el mismo conocimiento. Esta investigación implica una actitud constante de crítica y conciencia de los valores del investigador, posee un alto dominio de las aportaciones teóricas del entorno histórico en donde se producen los hechos

Por su parte Cook y Reichardt (1995) señalan que el investigador cualitativo, en lugar de utilizar definiciones operacionales, tiende a emplear conceptos y los significados de los acontecimientos, los mismos que aclaran las facetas múltiples del concepto.

Lo cierto es que los principios que guían las propuestas investigativas del siglo XXI son producto de la fusión paradigmática, disciplinaria y de la interpretación de la realidad social y de las dimensiones problemáticas en virtud de hacer frente y proponer soluciones.

Schwartz y Jacobs (1984) exponen la relación entre el conocimiento y el poder, es decir, la dificultad para reconstruir la realidad desde el punto de vista del actor. Ritzer, (1993) de acuerdo con factores pasados, explica el porqué de las acciones. Para Martínez (1999), la ciencia requiere, por lo tanto, de una profunda revisión epistemológica y metodológica 
en todos los niveles. Chávez (1996) señala que la fenomenología es una de las formas del humanismo neokantiano, en ocasiones llamado crítica por unos y hermenéutica por otros, la misma que surge en el siglo XIX en total oposición con el positivismo, y que disocia al mundo de las ciencias naturales y sociales. Colby (1996) señala que, dentro de la variedad de enfoques cualitativos, existe un común denominador que podría situarse en el patrón cultural, que parte de la premisa de que toda cultura o sistema social tiene un modo único para entender las cosas y eventos. Para Aguirre (1997), es un procedimiento que permite organizar diferentes tipos de datos en un marco de referencia o relación más coherente de manera que se puedan comparar y contrastar con mayor facilidad. Velasco y Díaz (1999: 220) afirman que "cuando el relato de unos hechos ordinarios y concretos condesan una visión relacional de valores y significados culturales, compuesta por el investigador, estamos ante una investigación densa". Por su parte, Rusque (1999) manifiesta que la fenomenología está fundada en una especie de intuición relacionada a la reflexión, al romper con las afirmaciones del positivismo que nos vincula con el mundo, y realizando abstracción de todo lo que el saber constituido a la ciencia pretende que aprendamos.

\section{EL PARADIGMA POSMODERNISTA}

A finales del siglo XX, los paradigmas posmodernos empleados son aquellos considerados necesarios y valiosos. Todo depende de que los hombres los reconozcan y acepten como tales. Se vive una época de pluralidad paradigmática, una etapa en la historia donde los investigadores emplean distintas formas de comprender los problemas surgidos de la realidad social y, a la vez, presentar soluciones a cada particularidad social en busca de la organización sinérgica a través de la diversidad, el cuestionamiento, y la libertad de pensamiento.

La nueva visión de la realidad de hoy se perfila en la comprensión de las relaciones y dependencias recíprocas y esenciales en todos los fenómenos: físicos, biológicos, psicológicos, sociales y culturales (Capra, 1992).

Para Webb (1996), la comprensión holística, "el todo y cada una de las sinergias están estrechamente ligados con interacciones constantes y paradójicas". Es decir cada acontecimiento se encuentra relacionado con otros acontecimientos, los mismos que producen entre sí nuevas relaciones en un proceso en el que se integra el todo.

El paradigma actual no existe como único y excluyente, tal y como lo afirma Martínez (1994), quien sostiene que "un nuevo paradigma exige el derrocamiento del anterior". En el año 2000, se afronta el crecimiento de distintos paradigmas emergentes regidos por la ciencia social, la filosofía, la sociología, la educación, entre otras ciencias paradigmáticas. El mismo autor en el 2004 señala que la metodología cualitativa no trata del estudio de cualidades separada, sino de un todo integrado que constituye una unidad de análisis, que estudia una cualidad específica. Esta aportación es corroborada 
por Hurtado y Toro (1988) al sostener que no existe un método único de investigación. Además, señalan que con la subjetividad se recurre a la comprensión intersubjetiva que viene tratando y exponiendo los alcances de estas metodologías cualitativas.

La investigación cualitativa encierra una serie de dificultades desde la perspectiva metodológica, porque los datos deben ofrecer la necesaria consistencia y emerger de una descripción y registro cuidadoso. El proceso de categorización juega un papel fundamental, ya que no se puede aspirar a una adecuada captación de la realidad en sus propios términos si no de logran elaborar las categorías que la hacen explicable y dan coherencia al flujo de eventos o conductas que se realizan en un contexto determinado. La metodología cualitativa no trata del estudio de cualidades separadas, sino de un todo integrado (holística) que constituye una unidad de análisis. Estudia la cualidad específica (Martínez, 2004). Por otra parte, Kerlinger y Lee (2002: 420) sostienen que "en la investigación no experimental no es posible manipular las variables o asignarlas aleatoriamente a los participantes o tratamientos". El tratamiento individual permite al investigador realizar la comprensión, interpretación y participación en forma personalizada.

\section{INVESTIGACIÓN CUALITATIVA}

La investigación cualitativa es aquella en la que se estudia la calidad de las actividades, relaciones, asuntos, medios, materiales o instrumentos en una determinada situación o problema. La misma procura lograr una descripción holística, esto es, intenta analizar exhaustivamente, con sumo detalle, un asunto o actividad en particular. La investigación cualitativa realiza registros narrativos de los fenómenos en contextos y situaciones e identifica la naturaleza profunda de la realidades (Fernández y Díaz, 2003). Es decir, la investigación cualitativa trata de identificar la naturaleza profunda de las realidades, su sistema de relaciones, su estructura dinámica. Los investigadores cualitativos hacen registros narrativos de los fenómenos que son estudiados mediante técnicas como la observación participante y las entrevistas semiestructuradas.

A este tipo de investigación le interesa lo particular, lo contextual, los relatos vividos, con predominio del método deductivo. Se adscriben a este enfoque, los estudios de casos, la investigación acción participativa, la investigación etnográfica, entre otros.

Para Bisquerra (1989), las características de la investigación cualitativa son las siguientes: el investigador es el instrumento de investigador, son estudios a pequeña escala en sus ambientes naturales, no pretende probar teorías o hipótesis, no tiene procedimientos previos de recojo de información, considera el fenómeno en su conjunto, todo el planteamiento de la investigación está sujeto a cambios durante la evolución y desarrollo, trata de categorizar la información y construir mapas significativos, normalmente no utiliza procedimientos estadísticos, puede incorporar elementos nuevos (no previstos), es detalla y documentada mediante notas o grabaciones, tal como se aprecia en la figura 1. 
Figura 1. Diferencias entre investigación cuantitativa y cualitativa.

\section{El ser humano y su relación con los enfoques: cuantitativo y cualitativo}

Enfoque cuantitativo

- Positivismo

- Hemisferio izquierdo

- Objetivo

- Método deductivo

- Análisis

- Criterios de fiabilidad

- Enfoque no holístico

- Paradigma racionalista

- Débiles en términos de validez interna

- Se limita a responder

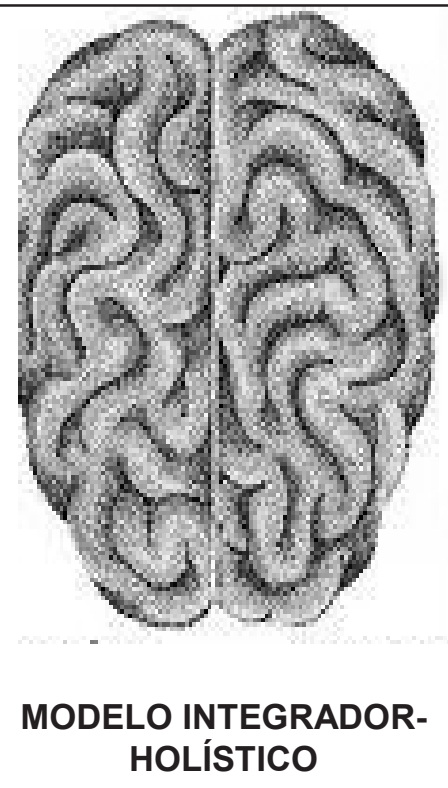

Enfoque cualitativo

- Fenomenológico

- Hemisferio derecho

- Subjetivo

- Método inductivo

- Interpretación

- Criterios de validez

- Enfoque holístico

- Paradigma naturalista

- Débiles en términos de validez externa

- Se limita a preguntar

Elaboración propia (Rivadeneira, 2013)

Morín (1981) afirma el problema del conocimiento es el corazón del problema de la vida. La persona que piensa contiene en una unidad dialéctica lo objetivo y lo subjetivo. El ser humano tiene dos mentes: la cabeza y el corazón, la interacción de las mismas permite al hombre pensar (objetivo) y sentir (subjetivo).

Alvira (1993) afirma que ambas perspectivas han llevado a cabo un proceso convergente y de reconocimiento mutuo. De hecho, la perspectiva cuantitativa ha aflojado en alguna medida cuando se refiere a la construcción de teorías a través de la modelización y simulación. Por otra parte, la perspectiva cualitativa ha seguido con su énfasis en la construcción y generación de teorías un aumento del aspecto verificativo y confirmativo. Por otra parte, Camphell (1974) sostiene que lo cuantitativo como cualitativo es un complemento indispensable e insustituible, y ha llegado a la conclusión de que "los metodólogos deben lograr una epistemología aplicada que integre ambas perspectivas".

\section{ENFOQUE CUALITATIVO}

Martínez (2004) sostiene que toda investigación, de cualquier enfoque, tiene dos centros básicos de actividad y estos consisten en: 
- Recoger toda la información necesaria y suficiente para alcanzar esos objetivos o solucionar ese problema.

- Estructurar esa información en un todo coherente y lógico, es decir, idear una estructura lógica, un modelo o una teoría que integre esa información.

Figura 2. Cualidades del enfoque cualitativo

- Presenta perspectiva humanista

- Hace énfasis en el lenguaje

- Interpreta los hechos humanos

- Propende a comunicarse con los demás

- Está limitado a preguntar

- Es débil en términos de validez externa

- Muestraun paradigmanaturalista:fenomenológico, comprende las pautas culturales y percepciones de sentido común a las situaciones concretas

- Tiene un enfoque holístico

- Presenta criterios de validez

- Requiere mayor cualificación teórica-conceptual al ser más difíciles y laboriosos

- Tiene perspectiva etnometodológicaMuestra comunicación horizontal

- Pregunta al enfoque cuantitativo ¿cuán particularizables son los hallazgos?

- Utiliza recolección de datos sin medición numérica para descubrir o afinar preguntas de investigación y puede o no probar las hipótesis en sus procesos de interpretación

Elaboración propia (Rivadeneira, 2012)

La investigación cualitativa estudia el todo en forma integrada, como una persona, una entidad etnia social, empresarial, etc., aunque se podría estudiar una cualidad específica, siempre que se tenga en cuenta los nexos y las relaciones que tiene con el todo. En fin, todos somos un "todo físico, químico, biológico, psicológico, social, cultural, espiritual". Por esto, el ser humano es la estructura dinámica o sistema integrado más complejo de todo cuanto existe en el universo (Martínez, 2006).

\section{MÉTODOS CUALITATIVOS}

El método Canon fue desarrollado por Kant y no se refiere tanto a lo metodológico, sino a los criterios de validez del conocimiento y a los procesos involucrados. El Canon apunta más a la interpretación y comprensión, y es el fundamento de técnicas tan variadas como la investigación, acción participativa, etnografía y la entrevista en profundidad. Este método dio origen a epistemologías tradicionales como la fenomenología y el humanismo. 
Es importante reconocer el método cualitativo que se empleará, es decir, dependerá de las naturales de estructura a estudiar.

Figura 3. Métodos de investigación cualitativa

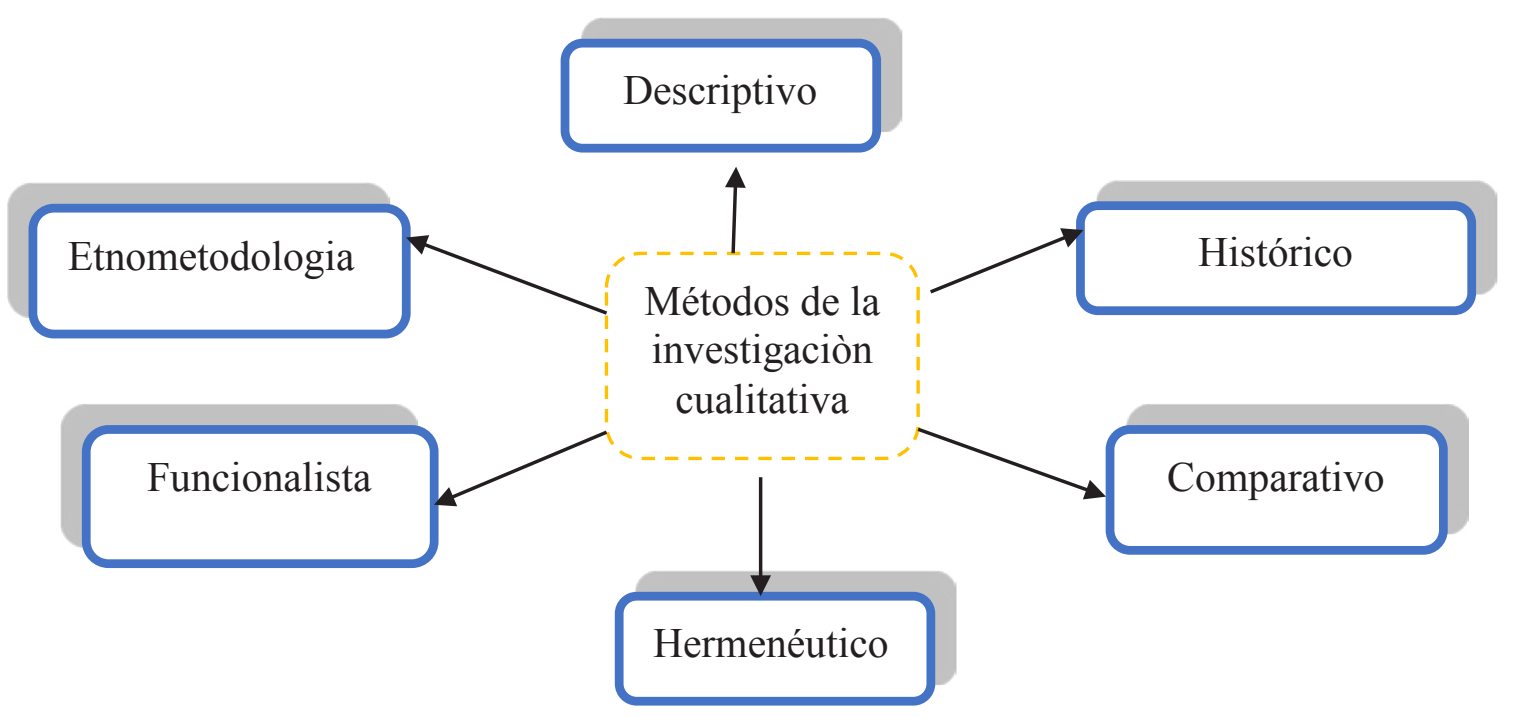

Elaboración propia (Rivadeneira, 2015)

\section{Método descriptivo}

Consiste en evaluar ciertas características de una situación particular en uno o más puntos del tiempo. Es una investigación que analizan los datos reunidos para describir así, cuales variables están relacionadas entre sí. La investigación descriptiva; describe una situación, fenómeno, proceso o hecho social para formular, a base de ello, hipótesis precisas. Los estudios descriptivos buscan especificar las propiedades, características y los perfiles importantes de personas, grupos, comunidades o cualquier fenómeno que se somete a una análisis (Danhke, 1989).

\section{Método histórico}

Estudia los eventos, procesos e instituciones de las civilizaciones, con el propósito de encontrar los orígenes o antecedentes de la vida social contemporánea y, de esta manera, comprender su naturaleza y funcionamiento. Se parte de la idea de que nuestras costumbres y formas de vida social tienen sus raíces en el pasado. a. Revisión de datos, b. Evaluación (o crítica) de los datos. c. Preparación de un informe escrito en el cual se presentan los hechos más notables

\section{Método comparativo}

Involucra la comparación de diferentes tipos o de institución o grupos de gente para analizar sus diferencias, así como sus similitudes. Se parte del supuesto de que estas divergencias y similitudes permiten conocimientos precisos de ciertos fenómenos, instituciones, estructuras y culturas. 


\section{Método hermenéutico}

Se refiere al intercambio recurrente entre observación, presunciones, interpretaciones tentativas, expectativas y verificaciones de datos nuevos y evaluaciones, que dan acceso a nuevas interpretaciones que corresponden a más datos.

\section{Método funcionalista}

Estudia las funciones sociales desde el punto de vista de las funciones que cumplen las instituciones o estructuras sociales. El funcionalismo afirma que el sistema total en una sociedad está interrelacionado e independiente, y que cada integrante cumple una función necesaria en la vida del grupo

\section{Método etnometodológico}

Hace hincapié en la interpretación y transformación de la realidad social, y en el actuar creativo de sujetos. El énfasis en los estudios etnometodológicos se centra en los acontecimientos cotidianos y las influencias del conocimiento común de las ciencias humanas. Para Coulon (1998), la etnometodología se centra en el estudio del proceso de una vida más razonable, es decir, de que los seres humanos no son meros seguidores de reglas ideales, sino que las modifican y transforman. Goetz y Lecompte (1988) coinciden con esta aportación.

\section{TIPOS DE INVESTIGACIÓN CUALITATIVA}

\section{Investigación participativa}

Geilfus (1997) señala que "todos participantes deben ser considerados como fuente de información y decisión para analizar los problemas y contribuir a soluciones a través de acciones conjuntas. Los principios epistemológicos de la investigación participativa, representan una propuesta metodológica para el cambio social. A continuación se menciona los siguientes principios: a. Consideración del mundo de distintas perspectivas. b. Consideración de la realidad de los integrantes del proceso. c. Tendencia de los sujetos a contextualizar su dimensión histórica. d. Construcción de la teoría a partir de la práctica. e. Participación popular que debe presentarse a través de todo proceso de investigaciónacción. f. Compromiso político e ideológico del investigador(a) con el sector popular y su causa. g. Reconocimiento del carácter político e ideológico de la actividad científica. h. Conversión de la investigación, educación y acción en momentos metodológicos de un solo proceso para la transformación social.

\section{Investigación etnográfica}

La etnografía ha sido concebida como la ciencia que estudia, describe y clasifica las culturas o pueblos. En el campo de la investigación socio-educativa, la etnografía constituye una alternativa metodológica que se diferencia de los métodos tradicionalmente empleados en este campo y de los métodos convencionales que por mucho tiempo fueron exclusivamente aceptados y recomendados para la investigación educativa.

Dentro de la investigación socio-educativa se puede decir que el término etnografía se ha utilizado para agrupar y etiquetar todos aquellos estudios descriptivos que dentro de 
la metodología cualitativa proporcionan una imagen de la vida, del quehacer de las acciones, de la cultura de grupos en escenarios específicos y contextualizados

El propósito fundamental de la investigación etnográfica puede ser solo describir una cultura o algún aspecto de una o más culturas en una organización. No obstante, existe una visión más amplia, compleja y relevante en cuanto al alcance de la metodología etnográfica. La investigación etnográfica permite reflexionar constante y profundamente sobre la realidad asignando significaciones a lo que se ve, se oye y se hace, desarrollando aproximaciones hipotéticas, redefiniéndolas continuamente, hasta llegar a construir e interpretar esa realidad sin anteponer el sistema de valores del investigador, lo cual conduce a la reconstrucción teórica.

\section{Investigación-acción}

El término investigación-acción (action research) fue propuesto por el psicólogo social Kurt (1946) para identificar una forma de práctica investigativa en la cual los grupos de personas organizan sus actividades con el objeto de mejorar sus condiciones de vida y aprender de su propia experiencia, atendiendo a valores y fines compartidos. Su modelo constituye un espiral permanente de reflexión y acción fundamentada en la unidad entre la práctica y el proceso investigativo, y se desarrolla a partir de dos ideas cruciales: la decisión del grupo y el compromiso con la mejora.

Kurt presenta cuatro fases del proceso: planificación, actuación, observación y reflexión. De este modo, una vez definida la preocupación temática del grupo (como área sustantiva en la que se centra la estrategia de mejoramiento), se elabora el plan de acción críticamente informado. Este es implementado, observándose los efectos de la acción, y se reflexiona colectivamente en torno a los resultados alcanzados, lo que conduce a una nueva planificación y al desarrollo de los ciclos sucesivos.

Nocedo y Cols (2009) citan quienes proponen una guía práctica de cuatro pasos:

\section{Paso 1.}

La decisión de dónde empezar es estratégica, por lo que es necesario examinar las condiciones objetivas y subjetivas existentes. El plan debe distinguir entre objetivos globales y objetivos estratégicos, así como entre objetivos a largo plazo y objetivos a corto plazo

\section{Paso 2.}

La puesta en práctica del plan y observación de cómo funciona la implementación debe tenerse en cuenta, ya que muchas veces al planear no se consideran todas las circunstancias, o estas pueden haber cambiado antes de empezar a actuar, lo que obliga a replanificar.

\section{Paso 3.}

El momento de la reflexión supone, analizar, sintetizar, interpretar, explicar y sacar conclusiones. Se revisa lo ocurrido y para lograrlo se integran las reflexiones en una 
exposición interpretativa que comporte conclusiones acerca de la preocupación temática, el plan inicial y lo que se ha aprendido en el primer paso de la acción.

\section{Paso 4.}

A partir de aquí se deducen implicaciones para el próximo paso, argumentando las mejoras que ahora se tratara de conseguir

\section{Estudio de casos}

El estudio de casos es una metodología de investigación ampliamente utilizada en el análisis de las organizaciones por las distintas disciplinas científicas (Hartley, 1994).

Existe la concepción de que los estudios de casos llevan demasiado tiempo para poder realizarse, y por lo tanto tienen como resultado documentos muy largos y difíciles de leer. El estudio de casos es una metodología amplia que utiliza técnicas tales como la observación, las entrevistas, los cuestionarios, el análisis de documentos. El estudio de casos no será definido por las técnicas utilizadas, sino por su orientación teórica y el énfasis en la comprensión de procesos dentro de sus contextos (Hartley, 1994).

Tradicionalmente los estudios de casos se han considerado como un método débil y carente de precisión, objetividad y rigor. Según Ruiz (1989), como método de investigación, tiene los objetivos siguientes:Evidenciar la experiencia interna de sí mismo desde una totalidad tanto en el tiempo como en el espacio.

- Captar la dinámica de la vida de la persona como un proceso vital y humano para descubrir los cambios y contradicciones por las que pasa.

- Interpretar la conducta del sujeto desde la visión de sí mismo, cómo ve el mundo y los demás que lo rodean, con vistas a encontrar las tendencias del sujeto en su entorno.

- Descubrir momentos claves de ciertos fenómenos de carácter general e histórico donde la experiencia y participación personal jugaron un rol.

- Durante todo el proceso, el investigador juega un rol activo, de actuación constante para poder profundizar en cada momento sobre aquellos aspectos que son esenciales para interpretar y comprometer la vida del sujeto. Así, es necesario tener en cuenta una serie de criterios o elementos esenciales:

- Ubicación contextual del sujeto en su cultura, medio económico, político, ideológico y social.

- Relación de los sujetos con otros miembros de su comunidad en la asimilación y trasmisión de la cultura.

- Valoración y acción social del sujeto sobre su realidad, mitos, ritos y experiencias básicas.

- Comparación de hechos y actividades a lo largo de diferentes momentos de su vida.

- Recreación del contexto social en que se da la vida del sujeto.

- Hechos significativos o determinantes en la orientación de la vida del sujeto. 
- Proceso de cambios que suceden a lo largo de su vida.

\section{METODOLOGÍA INTEGRADORA HOLÍSTICA PARA COM- PRENDER LA INVESTIGACIÓN CUALITATIVA}

La metodología integradora holística pretende superar cualquier disociación entre teoría y práctica. El propósito de la metodología no es descalificar los aportes teóricoprácticos de la investigación cuantitativa (Burrel y Morgan, 1997). Al contrario, pretende respetar las aportaciones en correspondencia con las contribuciones que se han venido dando al estudio del fenómeno. Para poder llevar a cabo este objetivo, el investigador debe hacer uso de las siguientes acciones metodológicas en el quehacer científico:

Acción 1. Relación entre el todo y las partes (deductivo-inductivo)

- Enfoque holístico: el ser humano-investigador: "Un todo físico, químico, biológico, psicológico, social, cultural, espiritual"

- Diálogo holístico: ser y la existencia

Acción 2. El estudio del fenómeno (análisis y síntesis)

- Recoger toda la información necesaria y suficiente para alcanzar esos objetivos, o solucionar ese problema.

- Estructurar esa información en un todo coherente y lógico, es decir, ideando una estructura lógica, un modelo o una teoría que integre esa información (Martínez, 2006). La investigación cualitativa y cuantitativa tiene como objetivo principal el estudio del fenómeno

Acción 3. Diálogo holístico (subjetivo-objetivo)

- La participación activa y democrática

- Respetar las dos grandes tradiciones filosóficas predominantes.

- Realismo e idealismo

Acción 4. Comprensión y explicación (interno y externo)

- Las dos cabezas:(pensamiento-sentimiento)

(Ver figura 4)

\section{CONCLUSIONES}

Una vez realizada la revisión de los lineamientos teóricos para cumplir con los objetivos que guían la presente investigación sobre comprensión teórica y proceso metodológico de la investigación cualitativa.

En relación al objetivo general: analizar los lineamientos teóricos que propician la comprensión del proceso evolutivo de la metodología de la investigación cualitativa. 
Constantemente se ha venido descalificando la validez de la investigación cualitativa, a pesar de los nuevos conocimientos que ha venido consiguiendo la ciencia mediante la investigación cualitativa. Solo se volvió la mirada a la investigación cualitativa cuando se amplió la comprensión de los aspectos psicológicos y socioculturales de la realidad y progresivamente se fueron legitimando sus técnicas y resultados. Destacando que muchas personas de formación positivista y de ciencias naturales se resisten a comprender el fundamento y las características de la investigación cualitativa. Para el desarrollo de esta investigación se ha realizado una revisión bibliográfica con la aportación de autores anglosajones, europeos y algunos de América Latina, la literatura denota la necesidad de establecer una metodología integradora holística que permita comprender el proceso evolutivo de la investigación cualitativa.

Figura 4. Metodología integradora holística

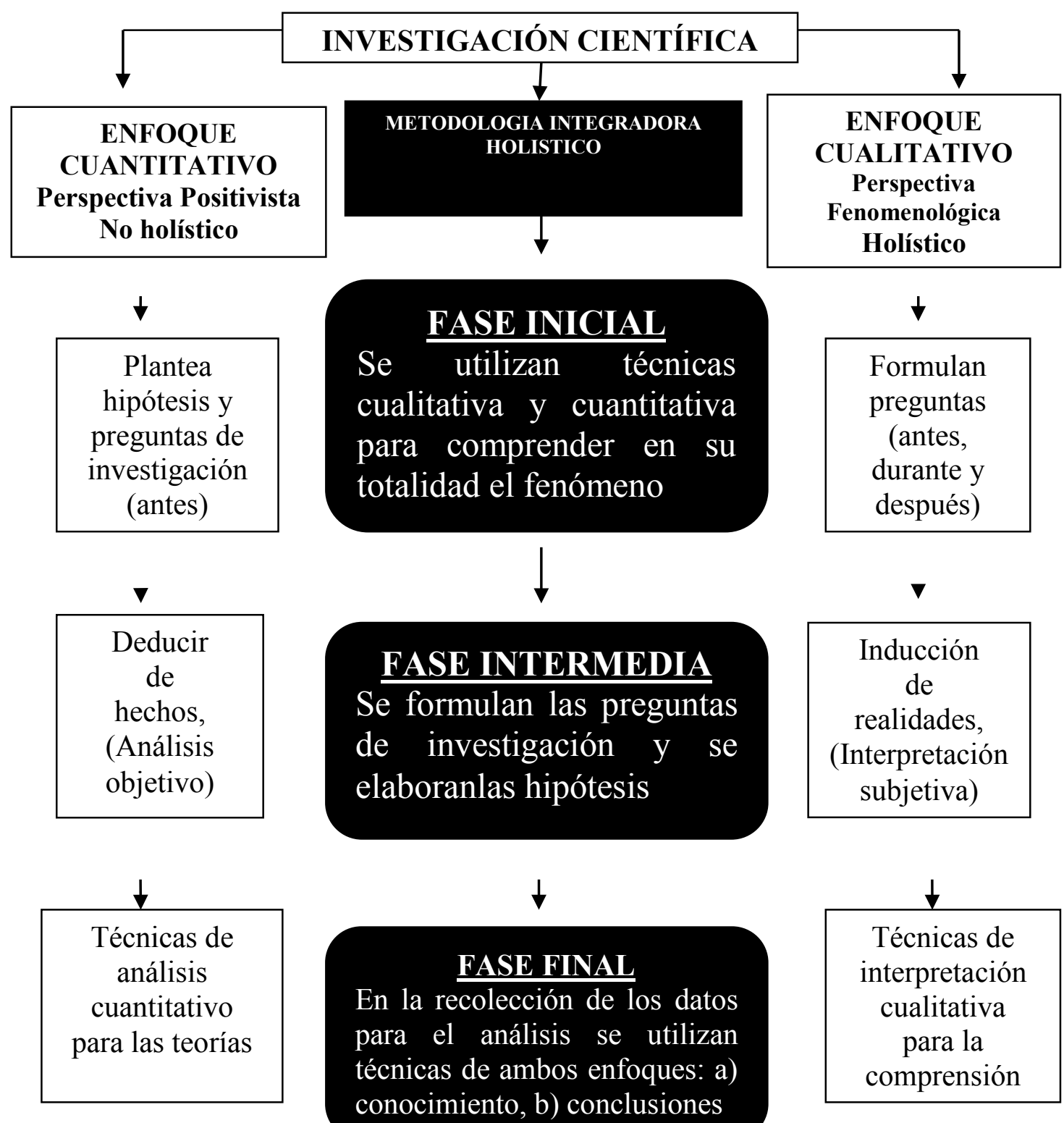


Elaboración propia (Rivadeneira, 2015)

En relación con el objetivo específico 1. "Revisar los lineamientos teóricos para garantizar la comprensión del proceso evolutivo de la metodología de la investigación cualitativa"

Esta investigación constantemente implica una actitud de crítica hacia los valores del investigador. Una visión nueva de la realidad invita a la comprensión de las relaciones y dependencias recíprocas en todos los fenómenos: físicos, biológicos, psicológicos, sociales, culturales

Con respecto al objetivo específico 2: "Explicar los lineamientos teóricos para garantizar el proceso evolutivo de la metodología investigación cualitativa"

Los métodos de la investigación cualitativa han sido usados tradicionalmente en la sociología y en la antropología social, también en áreas como la semiótica, la psicología, la educación y la historia, destacando además que al momento de llegar hacia el área de la salud con un enfoque positivista-experimental, la investigación es poco científica.

El objetivo específico 3 "Establecer las acciones de una metodología integradora holística apoyándose en los fundamentos teóricos y la comprensión para el estudio del fenómeno científico"

Para operacionalizar la metodología integradora holística se debe incluir las siguientes acciones:

- Acción 1. Relación entre el todo y las partes (deductivo-inductivo)

- Acción 2. El estudio del fenómeno (análisis y síntesis)

- Acción 3. Diálogo holístico (subjetivo-objetivo)

- Acción 4. Comprensión y explicación (externo-pensamiento) y (interno-sentimiento)

\section{REFERENCIAS BIBLIOGRÁFICAS}

Bisquera, R. (1989). Métodos de la investigación educativa. : Ed. CEAC.

Burrel, G. y Morgan, G. (1999). Paradigmas de la investigación social. Citados por Christian de Coch. Investigación cuali-cuantitativa en creatividad. España.

Campbell, D. (1974). Qualitative Knowing in Action Research. Communication to American Psychological Association.

Capra, F. (1992). El tao de la física. : Editorial Humanistas.

Chávez, J. (1996). La investigación educativa en América Latina. Habana: Ponencia para el Primer Taller en Metodología de la Investigación de la Facultad de Educación Infantil del ISP.

Cook, T y Reichardt, Ch. (1995). Métodos cuantitativos y cualitativos en investigación evaluativa. Morata. 
Coulon, A. (1988). La etnometodología. Ed. Cátedra.

Danhke, G. (1989). Investigación y comunicación. En Fernández Collado. Ciencia social.

Elliot, J. (1993). El cambio educativo desde la investigación-acción. Ed. Morata.

Fernández, P., y Díaz P. (2003). Ventajas e inconvenientes de métodos cuantitativos y no cuantitativos. En Cuadernos Monográficos Candidus 1 septiembre-diciembre.

Goetz, P. y LeCompte, D. (1988). La etnometodología. Ed. Morata.

Hartley, J. (1994): Case study methods. Sage Publications. : New bury Park.

Hurtado, J. y Toro, (1988). Paradigmas y métodos de investigación en tiempos de cambio. Editorial Episteme, Consultores Asociados.

Kemmis, S. y McTaggart, R. (1992). Cómo planear la investigación-acción. Ed. Liarte.

Kerlinger, F. y Lee, H. (2002). Investigación del comportamiento. Métodos de investigación en Ciencias Sociales. Interamericano.

Kurt, L. (1946). Action Research and Minority Problems. Journal of Social Issues, vol. 2, n. ${ }^{\circ}$ 4. Traducción de María Salazar, Universidad Nacional de Colombia (1990).

Martínez, M. (1994): Hacia un nuevo paradigma de la racionalidad. Caracas: Comportamiento.

Martínez, M. (1999). La nueva ciencia: Su desafío, lógica y método. Venezuela

Martínez, M. (2000). Análisis crítico de una metodología holística utópica. Artículo.

Martínez, M. (2004). Comportamiento humano. Nuevos métodos de investigación. Editorial Trillas.

Morín, E. (1981). El método I. La naturaleza de la naturaleza. España: Cátedra.

Nocedo, I.; Castellano, B.; García, G.; Addine, F.; González. C. (2009): Metodología de la investigación educacional. Ed. Pueblo y Educación.

Ritzer, G. (1993). Teoría sociológica clásica. España: McGraw- Hill.

Rivadeneira, E. (2013). Modelo investigativo integrado derivado de la investigación holística. Negotium, 26, vol. 9, septiembre-diciembre 2013, pp. 116-142.

Ruiz, J. (1989). La decodificación de la vida cotidiana. Método de la investigación cualitativa. España: Universidad de Deusto.

Rusque, (2000). De la diversidad a la unidad en la investigación cualitativa. Ed. Vadell.

Schawartz, H. y Jacobs, J. (1984). Sociología Cualitativa. Método para la reconstrucción de la realidad. Trillas.

Webb, E.; Campbell, D.; Schwartz, R., Secherest, L. (1966). Unobstrusive Measures Nonreactive research in the Social Sciencies. Chicago: Rand McNally.

Wilson, L. (1978). Seducción subliminal. Ed. Diana. Reimpresión 12a . 